\title{
BMJ Open The complexity of managing COPD exacerbations: a grounded theory study of European general practice
}

\author{
Mette Bech Risør, ${ }^{1}$ Mark Spigt, ${ }^{2} \mathrm{R}$ Iversen, ${ }^{1} \mathrm{M}$ Godycki-Cwirko, ${ }^{3} \mathrm{~N}$ Francis, ${ }^{4}$ \\ A Altiner, ${ }^{5}$ E Andreeva, ${ }^{6} \mathrm{~K} \mathrm{Kung}^{7}{ }^{7} \mathrm{H} \mathrm{Melbye}^{1}$
}

To cite: Risør MB, Spigt M, Iversen $\mathrm{R}$, et al. The complexity of managing COPD exacerbations: a grounded theory study of European general practice. BMJ Open 2013;3:e003861. doi:10.1136/bmjopen-2013003861

- Prepublication history and additional material for this paper is available online. To view these files please visit the journal online (http://dx.doi.org/10.1136/ bmjopen-2013-003861).

Received 26 August 2013 Revised 4 November 2013 Accepted 5 November 2013

CrossMark

For numbered affiliations see end of article.

Correspondence to M B Risør;

mette.bech@uit.no

\section{ABSTRACT}

Objectives: To understand the concerns and challenges faced by general practitioners (GPs) and respiratory physicians about primary care management of acute exacerbations in patients with chronic obstructive pulmonary disease (COPD).

Design: 21 focus group discussions (FGDs) were performed in seven countries with a Grounded Theory approach. Each country performed three rounds of FGDs.

Setting: Primary and secondary care in Norway, Germany, Wales, Poland, Russia, The Netherlands, China (Hong Kong).

Participants: 142 GPs and respiratory physicians were chosen to include urban and rural GPs as well as hospital-based and out patient-clinic respiratory physicians.

Results: Management of acute COPD exacerbations is dealt with within a scope of concerns. These concerns range from 'dealing with comorbidity' through 'having difficult patients' to 'confronting a hopeless disease'. The first concern displays medical uncertainty regarding diagnosis, medication and hospitalisation. These clinical processes become blurred by comorbidity and the social context of the patient. The second concern shows how patients receive the label 'difficult' exactly because they need complex attention, but even more because they are time consuming, do not take responsibility and are non-compliant. The third concern relates to the emotional reactions by the physicians when confronted with 'a hopeless disease' due to the fact that most of the patients do not improve and the treatment slows down the process at best. GPs and respiratory physicians balance these concerns with medical knowledge and practical, situational knowledge, trying to encompass the complexity of a medical condition.

Conclusions: Knowing the patient is essential when dealing with comorbidities as well as with difficult relations in the consultations on exacerbations. This study suggests that it is crucial to improve the collaboration between primary and secondary care, in terms of, for example, shared consultations and defined work tasks, which may enhance shared knowledge of patients, medical decision-making and improved management planning.

\section{Strengths and limitations of this study}

- The study brings forward shared concerns of managing chronic obstructive pulmonary disease (COPD) exacerbations at a cross-cultural level.

- Several local experiences exist but there is a lack of a general body of knowledge on challenges and solutions.

- Doing a cross-cultural study exhibits methodological limitations, eg how to take into account differing health system contexts.

- Focus group discussions (FGDs) do not tell us much about consultations as they are practiced but much about how they are thought to be practiced.

\section{INTRODUCTION}

It has been shown that many patients with chronic obstructive pulmonary disease (COPD) are diagnosed too late. ${ }^{1}$ Many patients who present to the emergency room with exacerbations have never been diagnosed with COPD. ${ }^{2}$ In addition, COPD is often misdiagnosed as asthma, leading to inappropriate treatment. ${ }^{3}{ }^{4}$ With regard to the management of COPD, much attention has been paid to the importance and difficulties of preventing and treating the exacerbations. ${ }^{5}$ According to GOLD 2013 the management of COPD exacerbations was included as a specific section on the combined management of COPD. ${ }^{6}$ Exacerbations lead to emergency and hospital care ${ }^{7}$ and each exacerbation leaves a permanent decrement of lung function. ${ }^{8}$ More patients need an expensive secondary care and long-term health status is hampered if management of exacerbations in primary care is suboptimal. ${ }^{9}$

To find novel solutions for improving COPD care, we need more research on experiences, practice and management approaches of the persons primarily involved in everyday care of COPD, the healthcare 
professionals and patients. A considerable amount of qualitative research on the needs and views of patients with COPD already exists. It has been shown, for example, that the uncertainty regarding the differentiation between asthma and COPD also has an impact on patients with COPD. In the early stages of COPD, patients do not recognise their symptoms, such as coughing, as the first stage of a severe disease. Consequently, they do not find their symptoms severe enough to warrant a physician's visit. ${ }^{10}$ Patients with COPD also often feel ashamed about their medical condition. They feel it is self-inflicted (caused by smoking) and the resulting shame is undoubtedly an obstacle in seeking medical advice, especially when they continue smoking. ${ }^{10} 11$ A previous research has shown that breathlessness is one of the most problematic symptoms of COPD. ${ }^{12}$ Good self-management with medication is very important for them to regain control of their breathlessness and lives ${ }^{11}{ }^{12}$; however, exercise programmes are approached with caution because of the breathlessness. ${ }^{12}$ Although patients report feeling confident about self-management of their medication, they are not confident about their actions in an emergency situation. ${ }^{11}$

Qualitative studies on healthcare professionals and their experiences of COPD treatment or care concentrated mainly on 'stop smoking' management. ${ }^{13-16}$ We have not found qualitative studies that investigated the views of healthcare professionals on regular COPD care, although a few studies focus on perceptions of end-of-life care, specific use of spirometry and underdiagnosis of COPD. ${ }^{17-19}$

There are large differences between countries in the way the primary care of patients with COPD is organised, and therefore local studies may have a limited generalisability. We wanted to know which experiences and challenges were shared by clinicians who care for patients with COPD. Therefore, we set out to investigate the experiences and opinions of GPs and respiratory physicians regarding COPD care in seven different countries (Norway, Russia, Germany, the Netherlands, Poland, Wales and Hong Kong). This analysis would lead to a clear understanding of the main concerns in COPD care. To investigate this in enough detail, we focused on the assessment and management of acute exacerbations. Our aim was to explore how GPs and respiratory physicians reason when managing patients with COPD exacerbations in clinical encounters.

\section{METHOD}

\section{Design}

The overall aim calls for a qualitative approach that encompasses a basic understanding of human interaction and social processes, for example, Grounded Theory (GT).$^{20}$ GT is furthermore, in its sampling and analytic approach, theory driven and strives for theory development of emerging categories. ${ }^{21}$ We employed a GT approach, which is mainly based on Charmaz's constructionist version, but we are also inspired by Corbin and Strauss' paradigmatic model of actions and interactions to help us develop an axis of the analysis. ${ }^{22} 23$

We chose focus group discussions (FGDs) as our data sampling method and designed a study of $3 \times 7$ FGDs. $^{24}{ }^{25}$ The study countries were selected from the start due to earlier research collaboration on respiratory diseases with networks (GRIN and GRACE): Wales, the Netherlands, Poland, Russia, Germany and Norway. The study originally was thought to be of only European, but early in the process we got an opportunity to include Hong Kong. Hong Kong was in the analysis primarily used for comparative purposes, adding an extra dimension to the analysis of the European countries. Each country performed three FGDs with new participants each time: FGD 1 with only general practitioners, FGD 2 with only respiratory physicians and FGD 3 with a mix of GPs and respiratory physicians. The first FGDs were undertaken in March 2011 followed by FGDs 2 in September/October 2011 and FGDs 3 in February/ March 2012. All researchers from each country who were responsible for conducting the FGDs participated in a 3-day workshop where they were taught the methodology of GT and FGD methods, theoretically and with practical exercises. This was performed in order to streamline the methods across countries and try to secure the shared knowledge and practice of the methodology, basic to making a cross-country analysis.

All countries used the same topic guide each time with a selection of already formulated prompts. Before conducting FGD 1 the first topic guide was developed by the main author with input from collaborating countries. A pilot interview was conducted in Tromsø in order to adjust for formulations, phrases and questions. Between FGDs 1 and 2 the topic guide was discussed and revised among the same authors but also with input from research leaders from the other countries-all met for a 1-day workshop to discuss the categories identified in a preliminary analysis of FGD 1 and to decide on how to sample data according to this, that is, whom to include in FGD 2 and what to ask. Between FGDs 2 and 3 a major revision of the topic guide was made, also after a preliminary analysis and development of categories and concepts, but this time input was made by email from most countries apart from the researchers in Norway and the Netherlands who met to discuss the revision. This last topic guide focused especially on providing knowledge on topics that were still unclear but also on solutions to identified challenges and difficulties on collaboration. The two first topic guides included three patients' cases (see online supplementary files) to prompt the discussion on the first three topics. These were not a part of FGD 3 but instead the topics and the results from FGDs 1 and 2 were elaborated into new questions.

The interview guides contained the listed topics, based on the known issues of concern to GPs. The respiratory physicians were asked to discuss the routines 
in general practice as it was known to them, but inviting them to be open about their own views and concerns. The topic of collaboration was mainly brought into FGD 2 to try to meet a mutual concern, and the discussion resulted in change of the third FGD's interview guide:

FGD 1: assessment and medication/hospitalisation/ self-treatment/use of guidelines/challenging or difficult situations/most important problem/improvement of clinical practice.

FGD 2: assessment and medication/hospitalisation/ self-treatment/use of guidelines/challenging or difficult situations/most important problem/improvement of clinical practice/collaboration with GPs (the topics in FGD2 were supplemented with more specific subquestions than in FGD 1).

FGD 3: diagnosis of exacerbation/hospitalisation of borderline cases/criteria for self-treatment/collaboration between primary and secondary sector/who are the difficult patients (the topics of FGD 3 were supplemented with subquestions aiming at describing bottlenecks and future solutions for each topic).

\section{Sampling and material}

All 21 FGDs were performed with a moderator and an assistant moderator, except for a few where only a moderator was present. The moderators were the head researchers from each country network (a professor or doctor in family medicine), and in one case a $\mathrm{PhD}$ student and an epidemiologist. They were all closely supervised. The head researchers had their main research field in respiratory diseases in general practice and the epidemiologist had a long experience with intervention research in general practice while the $\mathrm{PhD}$ student was new to the research field. Comoderators were either skilled qualitative researchers or GPs with research experience. The aim was to include $6-8$ participants for each FGD.

Participants for FGD 1 were GPs and were sampled purposefully to cover rural and urban practices. They were invited via an information letter. Several information channels were used in some countries, making contacts via meetings, health boards, email, telephone, mail or personal contact. By the end we had between 6 and 10 participants in each FGD and these lasted between 1 and $2 \mathrm{~h}$. Several GPs did not wish to participate due to limitation in time and interest.

Participants for FGD 2 were sampled among hospitalbased respiratory physicians and private or out-patient respiratory physicians, depending on the specific health system in each country. The number of participants varied between 4 and 7 and the FGD lasted around $1.5 \mathrm{~h}$. The participants were found via email invitations, personal contacts or at hospital wards (eg, having a respiratory physician ask colleagues) and sampling was faster and easier than in FGD 1 but also resulted in fewer participants.

Participants for FGD 3 were sampled among GPs and respiratory physicians to enhance a discussion on the emerging analytic categories in the data. FGD 3 had between 6 and 9 participants and lasted from 1.5 to $2 \mathrm{~h}$. The sampling took place via e-mail invitations, through personal contact or inviting a key person to ask colleagues. The composition of the FGDs had a balanced number of GPs and respiratory physicians.

In some cases, the GPs came from the same practice and knew each other. Also, respiratory physicians were acquainted with each other, working at the same hospital or out-patient clinic. In some FGDs the moderators knew a few of the participants but this was not a dominant tendency. The participants were not sampled according to gender or seniority but we intended to include physicians working in rural and urban settings as well as at different types of health workplaces, mainly regarding the respiratory physicians as aforementioned. The ones who were included and informed us about their practice time, about half of the participants, showed a long average seniority (approximately 14 years) with the respiratory physicians having the most seniority. GPs practiced in single clinics, shared clinics and group practices as well as in health centers, and respiratory physicians practiced at hospitals (regional as well as university hospitals), outpatient clinics and in several cases at both places.

All FGDs were performed within the university premises and they were transcribed verbatim from audio recording by the local researchers and translated into English by a skilled translator.

\section{Analysis}

The analysis took place according to GT methodology from the first round of FGDs, using Nvivo V. 9. First and third authors did the main coding, that is, line-by-line coding in the beginning but we also coded by event, constantly looking for meaningful categories and concepts in the data and making comparisons across all FGDs. The first-round analysis of FGD 1 was performed by the Troms $\varnothing$ team mainly and its purpose was to guide us and decide which categories and concepts should be elaborated or perhaps de-emphasised in the following FGD. The same process took place between FGDs 2 and 3. This whole approach aimed for a process of theoretical sampling. Furthermore, the analysis also contained attempts to conduct focused coding ${ }^{23}$ in order to find the most significant categories, concepts and actions of the material, and this was again extended with a preliminary axial coding trying to structure a relation between categories and subcategories. ${ }^{22}$ The axial coding was elaborated further in the final analysis, and this was supplemented with a paradigmatic matrix that organises data into conditions/actions and interactions/ consequences and helps to develop categories and find relations between them. ${ }^{22}$ This paradigm also highlights the actions and interactions of actors involved and helps to define the core categories of actions/social processes with their related dimensions, which finally are developed into a grounded theory. A special emphasis is put 
here on intentions and goals of the actors in the process. Memos were written especially in the last phase of the analysis, giving a solid basis to start comprehending the main concerns for the health professionals concerning COPD exacerbations and how they handled them. The analysis made by the Tromsø team was supplemented and discussed with input from the study countries' teams. This created collaboration in terms of the analysis and strengthened the validity of the findings. The analytic findings are presented below in a structure which first delivers the interpretation of findings and subfindings, second illustrates these with quotations and third, in some cases, elaborates or summarises the interpretation based on inferences from the quotations and on the overall analytic perspective.

\section{RESULTS}

\section{Balancing management within a scope of concerns}

Overall, data displayed several distinct discussions on how to manage COPD exacerbations. Some FGDs were very focused on problematic issues from the beginning, others seemed more straightforward and practical, not paying much attention to any difficulties, while others again from the beginning tuned in on uncertainty concerning medical practice and knowledge, prone to discuss uncertainties and doubts. Some, after prompting by a patient history, were dominated by a demonstration of medical knowledge at the start. However, all FGDs combined their medical concerns and discussions with attempts to describe and understand the patient's social circumstances, as well as to take into account the particular health system and its resources as determining factors in the management of exacerbations. When it came to discussions on main challenges, clinical practice and collaboration, several societal problems and political dimensions of health work were addressed, for example, with a tendency to shift the level of attention from biomedicine to health promotion, health services topics and general health population issues.

The analysis of all FGDs made it evident that the management of COPD, especially of exacerbations, is experienced as trying to balance between medical knowledge and practical, situational knowledge. Balancing management per se is the main concern for GPs and respiratory physicians, resolved mainly by strategies of knowing patients, their social resources and health contexts. However, this concern only makes sense when subcategories of concerns are explained and analysed one by one, showing the mechanisms of an interconnected process, that is, a scope of concerns.

\section{Dealing with comorbidity}

'Dealing with comorbidity' is a concern which is significant for three different dimensions of clinical management of exacerbations: how to be sure it is an exacerbation, when to prescribe antibiotics or steroids and when or whom to hospitalise. Comorbidity refers here to the existence of disease conditions other than COPD, such as asthma, cardiovascular disease, skeletal muscle dysfunction, metabolic syndrome, osteoporosis, depression and lung cancer.

Crucial to a diagnosis of exacerbation, we found in the FGDs that all the physicians wished to see the patient when he/she calls the GP and asks for antibiotics because of a worsened condition. To merely give advice or to prescribe over the phone could be suitable for some, but only if they knew the patient well. As such, making a diagnosis of COPD exacerbations was closely connected to seeing, knowing and examining a patient. However, just as important, symptoms like breathlessness and anxiety were understood to be caused by multiple possible diagnoses, that is, the questions of comorbidity blurred the picture. Not everything resembling an exacerbation is one:

It is difficult, for example, to diagnose COPD in an 80-year old patient. I had such a patient. The doctor [GP] diagnosed her with asthma. This is a non-smoking patient. Her FEV1 is $27 \%$. I have a question: what is it? Is it COPD or asthma? The test with bronchodilator was positive. Such mixed cases are difficult. Although today we treat such cases in the same way, with combined medicines. In general, we have a lot of difficulties in diagnosing the severe patients. (Russia FGD 2)

In terms of theoretical medical knowledge, making a diagnosis could be presented by the physicians as straight forward, but the clinical picture of comorbidity confused the process:

Yes, but still, it [making a diagnosis] is not difficult at all. It's the matter of, it's the many comorbidities among them, everyone at risk for COPD is rather at risk of, well, cancer and cardiac diseases are quite common among COPD patients. (Norway FGD 3)

Diagnosing was perceived overall to be a clinical process made over time, using in particular the patient's history and experiences to estimate an exacerbation, trying to judge if the patient does have an exacerbation or not.

Comorbidity was also described as a crucial factor when deciding whether or not to prescribe antibiotics and steroids, and this made prescribing decisions difficult:

these patients have often got a load of other things wrong with them, they are high risk individuals, they have been smoking for many years and I think the main worry is about, is the things that we talked about, is multiple pathologies, multiple drugs. (Wales FGD 1)

Concerning the steroids, the respiratory physicians in FGD 2 seemed to be, overall, in favour of prescribing steroids for an exacerbation for a limited time period and discussed more intensely whether to give antibiotics and on what basis, due to resistance considerations. Conversely, for the GPs it was 'easier' to prescribe 
antibiotics, but giving both drugs at the same time was not their first option, especially when considering comorbidity. The side effects of long-term use of steroids and the risk of inducing pneumonia were decisive issues for the GPs:

Usually, we should be concerned whether there is an infective component when we prescribe steroids. Usually chronic COPD patients are weak and if we prescribe steroid for them, it will be easy for them to get infection and often I will prescribe antibiotics as daily practice. (Hong Kong FGD 1)

The comorbidity is high and I reckon that every COPD patient has some form of presence of one or more disorders in addition to a primary disease or disorder... [...]... When visiting the practice I will often put them on a scale. They need to be weighed anyway for COPD. So if they have gained $4 \mathrm{~kg}$ I am not sure to send them home with a dosage of prednisone and say they'll be fine. (The Netherlands FGD 1)

When considering whether or not to hospitalise, this was illustrated in long debates on pros and cons. The possibility of comorbidities such as heart failure, diabetes, pneumonia, anxiety or psychosomatic disorder was an important consideration for the physicians. A suspicion that a patient had an undiagnosed comorbidity might be reason enough to admit a patient.

E: I think it also depends on the severity of the disease before the exacerbation, that is, how much strength the patient has got left. If he is unstable anyway, even if he isn't exacerbated, then that's a reason to hospitalize him of course. The other possibility would of course be, that you have been treating him with different therapies and you say, I don't see any significant changes, and this has to be analysed in more detail.

G: And it also depends on the comorbidities, what other diseases he has. (Germany FGD 3)

For COPD exacerbations in general objective assessments, such as low oxygen saturation and rapid respiratory rate, were the most obvious criteria for hospitalisation:

I think there are two important things with this case, firstly how well the GP knows the patient so he can compare them to their baseline and secondly an objective assessment, oxygen saturations, respiratory rate, heart rate, all these sort of things, a clinical consultation, I think. (Wales FGD 2)

On the other hand, after taking into account the patient's basic medical status, how well the patient is known and his general condition, decisions about hospitalisation came down to whether one would risk letting the patient stay at home despite comorbidities or social situation, or wishes:
[I will assess]...age, comorbidity, the increase of respiratory failure, whether a patient can sleep or he is sitting all night in this position, orthopnea as he can... what kind of work around the house, he can perform. Can he dress himself, can he move around the apartment, or does it gives him a hard... can I manage treatment... And if relatives can help in treating him at home... (Russia FGD 1)

On the other hand you also have to see, how multimorbid the patients are. Cardiac decompensation, exacerbated COPD, back and forth again and again, sure it's difficult, but you have to ask yourself "how far can I treat this locally? What can I achieve?" So that we have, right, we all also are in the ambulatory area, we also have our limits. And if we have such complicated cases, where there is something wrong again and again, and we have to ask us diagnostically "What is the real problem?", there I sometimes believe, that some patients, also if they are tired of it, would be better off at a hospital. (Germany FGD 3)

Yes, and so social factors also play a role, if she lives alone and doesn't have any security network at home you can, at any rate, be more unsafe when deciding to send her home, and I assume that one would tell her, in case you don't admit her... you would have a lower threshold to do so if she gets worse. (Norway FGD 2)

The elements of medical decision-making, that is assessment, tests, diagnosis, medication, hospitalisation and overall management, were on one hand discussed within the framework of highly complex medical matters, weighing biomedical, pharmaceutical and technological knowledge against each other, and on the other hand taking into account knowledge of the social context of the patient. Indeed, the patient's social condition, background, resources and personal profile were highly important for all concerns of 'dealing with comorbidity' and clinical decision-making in order to balance medical knowledge:

There are so many things that you have got to take into account like social circumstances, does he have someone in the house to keep an eye on him, do they have a whole load of other co-existing illnesses, um you know it's very difficult to pick on one thing, it's you trying to make a decision on a number of factors. (Wales FGD 1)

Participants' thoughts about the use of medication indicated a specific concern for the patient and a wish to know more to support a medical decision. These thoughts were medically informed and patient-centred, for example, how high doses were acceptable and for how long, when was it rational to prescribe antibiotics or steroids according to clinical findings and history, how would the patient accept medication, would he be compliant (concerning up-take and purchase of medicine) and would he be able to have a dialogue on effects and use with his physician: 
Now we talk a lot, but if you know him, then you know whether he already had something like this in the past. Do you have the case history, does he have compliance? Does he take his medication? Or not? Or is he someone who comes along, I don't know, and maybe had an infection as a trigger, he still smokes heavily and now found his spray and has been using it for 2 days and it doesn't help yet. (Germany FGD 1)

Thus, knowing the patient in several ways and at several levels was a common approach, and this situational knowledge was used for gaining a complementary understanding of how to treat the patient and determine the necessary medical steps to take.

\section{Having difficult patients}

The second concern emerging from the FGDs is 'having difficult patients'. This concern contains a shift in perspective moving from seeing 'comorbidity' as a problem to how the GPs and respiratory physicians may see the patient and the relationship with the patient as a problem. In this move, the patient as a person and his/ her personality, situation and context are in the foreground, not the medical condition per se.

A common dimension of 'the difficult patient' is presented in some of the first reactions from the GPs and respiratory physicians when they start talking about patients with COPD. On one hand, it is a relief that the management and treatment of these patients in general has become easier and more successful due to new drugs (although treatment may still be complicated because of complex conditions), but, on the other hand, some physicians feel annoyance when they perceive that patients have unrealistic expectations:

In general, these patients are more difficult; the patients, who have a lot of expectations, and believe that all of their 'affairs' will be resolved for them (by someone else). I don't particularly like those patients; they are difficult to cooperate with, perhaps, just because of their belief that everything should be done for them. (Poland FGD 1)

More often though, having patients with COPD exacerbations generates mixed feelings of concern for the patient. The patients are difficult exactly because they are seriously ill and suffer and need complex attention, while at the same time they are difficult because they are time-consuming, frequent attenders, noncompliant and often do not give the physician the satisfaction of being helpful:

As for me COPD patients are men with long smoking experience, they are often poor, of low social status, alcohol dependent, they usually don't follow the prescribed treatment. They simply don't have enough money for treatment. I examine them and usually refer to the hospital or to the expert bureau. Generally it is a very sad story, usually leading to disability. There are very few effectively treated patients. (Russia FGD 3)
Especially, when discussing self-treatment, the patient and his/her social profile turn up as a problem-probably because here the patient is delegated an active role, and behaviour and context becomes even more decisive for a decision to prescribe self-medication. Being a 'difficult patient' is further dependent on a spectrum of difficulties pertaining to the patient himself, such as poor illness perception and understanding (resulting in undertreatment or overtreatment), smoking habits/lifestyle/behaviour, poor intelligence, poor compliance, old age and bad quality of life/poor economy. But the label of being difficult also results from unsuccessful/lack of interaction and communication with the health professionals to improve one's situation together.

There are a couple of things we encounter such as most patients are 'dead horses'. This does not sound respectful but there are a lot of patients who want to be left alone. We cannot make them understand what we expect from them. Be active, quit smoking, more exercise, loyal to therapy, take their own initiatives. (The Netherlands FGD 2)

We refer them to this school while they are on a sick leave. We try to convince them of something, especially of the necessity to refuse smoking. It seems that they agree, nod, everything is understood, but they don't come to the second or third meeting. We don't see the light in their eyes; we don't see their initiatives, their participations, any support for their care. Therefore, we have refused to conduct school for COPD patients as we don't see the interest from the patients. (Russia FGD 2)

In other words, a patient was seen as 'difficult' as a result of an interaction, or 'difficult' was an already made characteristic clinging to certain disease profiles, either due to earlier experience with a patient or due to generalised knowledge of patients with COPD. Moreover, the difficult patient is a typology referring to the continuum of concerns and not all patients are labelled as such. Some, indeed, are mainly difficult because they have difficult illness conditions. But still, the physicians' perceptions of having 'difficult' patients in terms of social and personality-related difficulties, not explicitly related to the medical condition, govern several interactions. The physicians try out several practical solutions to this and one important overall action suggested is to get better at motivating the patients. Motivation is the key to several aspects of the difficulties they meet in patients. A repeated advice here is to teach, instruct and inform patients-about the disease, about medicine and especially about the right self-treatment. To teach and motivate is the main action pertaining to 'the difficult patient'-but it builds on the necessity to know and involve the patient in a relationship in order to address him/her properly:

I think that is what GPs are supposed to do. I think that most GPs, especially the younger ones, think highly of communication. It matches well. It is difficult and the 
relationship between yourself and the patient is very important. Getting people to quit smoking. The times that you succeed are very rewarding. People are genuinely happier/healthier when they have quit smoking. And the whole story of empowerment as they call it, that you trust the patient to be able to handle his/her own problems. I think that patients are rather dependent. (The Netherlands FGD 1)

\section{Confronting a hopeless disease}

The third concern in the scope of management deals with how to balance one's approach to a disease that confronts the physician with his medical professional limits, that is, the limits for curing and saving lives, and with the patient's existential deterioration at all stagessuffering in general and at end-of-life stages. In this approach, it is primarily the disease in itself which the physicians react emotionally upon. It is the disease that makes the encounter with the patient becomes characterised by shifting feelings of empathy, hopelessness or frustration, notably related to COPD in general more than to only exacerbations. The severity and poor prognosis of the disease per se gave, for example, either an atmosphere of frustration or simply created a pragmatic attitude:

They are the most severe patients among the patients with broncho-pulmonary pathology. This is the category of severe patients, which you don't know how to help, in spite of all the standards what exist today. Dyspnea will come anyway. Neither oxygen, nor steroid therapy, neither bronchodilators, nor courses of antibioticssometimes nothing for these patients can be done if a patient has severe COPD. (Russia FGD 2)

Another difficult matter is that they don't get better at all...[...]... it's like you prescribe one drug after the other but their conditions worsens gradually and they suffer from difficulties in breathing and in the end we have nothing left to help them with. (Norway FGD 1)

Other reactions seen were expressions of sympathy and feeling sorry for the patient in the light of a quickly deteriorating chronic disease:

And you also have these fatal developments, we know that about COPD, you can use the maximum therapy and the patients do as they are told, but still it gets worse bit by bit. And that's especially dire. That's where you really pity them, because you are so powerless. That's how it is then. (Germany FGD 3)

Another often mentioned dimension of this concern dealt with smoking. Smoking habits and the failure of smoking cessation were considered as the main obstacles for the prevention of exacerbations and the discussions on this topic were often marked by hopelessness concerning the patient's capabilities of cessation.

We give patients with mild or moderate stages of COPD anticholinergics, explain them why they need such treatment. And they do not give up smoking. Sometimes I have the opportunity for a whole hour to talk with the patient. [We] spend a lot of energy and the energy without any feedback. Dim eyes. [They] like smoking, and continue smoking, although they agreed to stop. But he doesn't do anything. (Russia FGD 2)

Continuous smoking habits became unintelligible to the physician and his attempts to ease symptoms and relieve a condition, especially when a patient experienced acute exacerbation. Patient's smoking habits in general were an especially dominant concern for the physicians, not merely as a simple lifestyle issue. They invoked strong emotional reactions among the physicians when faced with suffering, deterioration and death due to smoking. In the light of this, many discussions on smoking cessation may be seen as attempts to strategically manage a critical disease and an experience of being professionally helpless and emotionally touched. Also non-compliance of medication was a source of discontent and puzzlement among the physicians. Such experiences often created an air of hopelessness and helplessness which were related to the concern of 'having difficult patients'. But here we wish to emphasise that the feelings of the physicians were triggered by the disease more than by the interaction with the patient.

The feeling of hopelessness was experienced also in relation to systemic factors; for example, if oxygen was not available or hospital care was inadequate. Despite all these negative experiences, an overall preoccupation with the patient was always present, resulting in actions of care even for the patient with 'the hopeless disease'. For those who might still increase their quality of life, discussions on the value of rehabilitation and especially physical exercise came up as an answer and as possible strategies to prevent deterioration into stage III or IV COPD. Rehabilitation was especially brought up as an overall concern for the patients' social life, involving several suggestions of practical advice and how to teach patients and their families, for example, basic disease management, physical exercise and smoking cessation, organised in COPD schools. However, rehabilitation attempts were hampered by a lack of programmes, access, financial priority and collaboration, especially in Russia and Poland.

It is not being founded. Anyway, there is no tradition of rehabilitation in diseases of the respiratory tract in Poland. (Poland FGD 2)

...no one said anything about the rehabilitation of patients... here it is, I think quite an important point... and, by and large, we do not know how to perform [rehabilitation]. (Russia FGD 1)

... the evidence is very clear, that a rehabilitation program on COPD-exacerbation is something extremely good. The evidence is OVERWHELMING and the health insurance companies nearly NEVER cover the costs for it. 
And that's something that can drive you mad. That the evidence is crystal clear, but the attitude of the insurance is also crystal clear: We won't finance rehabilitation programs. And that is a daily conflict that we have to fight. (Germany FGD 2)

But, but that is the solution, the solution is not to send patients to an extremely expensive rehab center, the solution is gathering people, making people aware, and I am convinced if you buy a set of Nordic walking equipment and you find two buddies to walk with, you have both the element of resocialising and a healthy exercise combined, people are given perspectives again, for 30 euros you can set up an exercise program. Thirty euros for Nordic walking equipment, when buying at Aldi it is even cheaper. But what you need is to make the patients aware of it, and when they do not concretize and you will treat them with drugs it will be ineffective. The challenge and art is to motivate the patient, and subsequently imbed Nordic walking equipment. (Netherland FGD 2)

Palliation for patients with COPD came up as a topic of discussion in many FGDs. This was phrased as a worry that the patients would not receive the optimal palliative attention, for example, compared with patients with cancer, or that the physicians did not attend enough to provide palliative care:

....... ...not going to make them better, so a lot of them will just slowly progress and they'll get worse and worse and worse and then eventually what we should be doing is referring a lot of them to [palliative care], they shouldn't be down the chest side of things and so, palliative care deals with cancer that's fine, you know, but they don't deal with conditions that aren't cancer, no, no they do, they do but what I'm saying is that now we should be using that a lot more. (Wales FGD 2)

\section{DISCUSSION}

The management of acute COPD exacerbations was dealt with within a scope of concerns. These concerns ranged from 'dealing with comorbidity' through 'having difficult patients' to 'confronting a hopeless disease'. The first concern relates to medical uncertainty regarding diagnosis, medication and hospitalisation. Here, the clinical process was often presented as straight forward in terms of theoretical medical knowledge, but became blurred by issues of comorbidity and social context. The second concern is when 'difficult' becomes an attribute of a patient. Patients were difficult exactly because they needed complex attention, but even more because of personality aspects triggered annoyance, they presented poor illness understanding, and were time consuming, did not take responsibility and were non-compliant. The third concern relates to the emotional reactions by the physicians when confronted with 'a hopeless disease' due to the fact that the disease is chronic and progressive and the treatment options slow down the process at best. Physicians met their own limitations and reacted to end-of-life stages of COPD and patient's poor quality of life. GPs and respiratory physicians balance the concerns of 'dealing with comorbidity', 'having difficult patients' and 'confronting a hopeless disease' with medical knowledge and practical situational knowledge, trying to encompass the complexity of a medical condition. They engage vividly in suggestions to improve future consultations and patient lives, making an effort to create effective medical routines (see figure 1).

Comorbidity and the social context of the patient complicated the management of disease. In everyday practice the complicated rather than the straight-forward patient profile is probably most common, considering the high prevalence of comorbidity in this patient group. ${ }^{26}$ This also illustrates that physicians feel the lack of good guidelines incorporating comorbidity issues clearly. $^{27}$ Therefore, an important aim for future COPD guidelines would be to describe management options within the context of the most prevalent comorbidities in COPD. In addition, there is a need for more pragmatic trials in patients with COPD that do not exclude elderly patients or patients with comorbidity. Increased collaboration between general practice and hospitals was also suggested as an approach in dealing with uncertainties around comorbidities. Collaboration could lead to standardisation of assessment, establishment of joint consultations in order to make pulmonology services more available to GPs, definition of work tasks specific for each specialty and more involvement of GPs in hospitalisation decisions and discharge.

Patients with COPD who experience exacerbations are commonly viewed as difficult patients, with some physicians even saying that they do not particularly like these patients. Little is known about this emotion among physicians, but this finding is in agreement with qualitative studies among patients with COPD where they express the feeling that they are blamed for their self-inflicted disease, not only by their own social environment, but also by healthcare workers. ${ }^{28-30}$ The management of COPD exacerbations and stopping smoking require an active role of the patient, so it is understandable that healthcare professionals may feel frustration if their advice is not followed. On the other hand, the resulting emotional impact of blaming patients is likely to have a negative effect on the patient's mood, which will further hamper the relationship and the clinical process. ${ }^{30}$ The 'difficult' patient is well known from studies on other kinds of patients and is commonly attributed by physicians to mental disorders, personality traits or morally flawed behaviour. ${ }^{31}$ Fiester, however, argues, that the label 'difficult' is best explained by problematic interactions or reactions to the delivery of care, and he also notes, similar to what we found, that a physician may label a patient 'difficult' or 'hopeless' because of her or his own inability to effectively diagnose or treat the problem or because of a patient's reaction to this failure. He points to the problem as an ethical one requiring an ethical consultation service. In our study, to overcome 'difficult', the physicians focus on improving 
Figure 1 A grounded theory of chronic obstructive pulmonary disease exacerbation management.

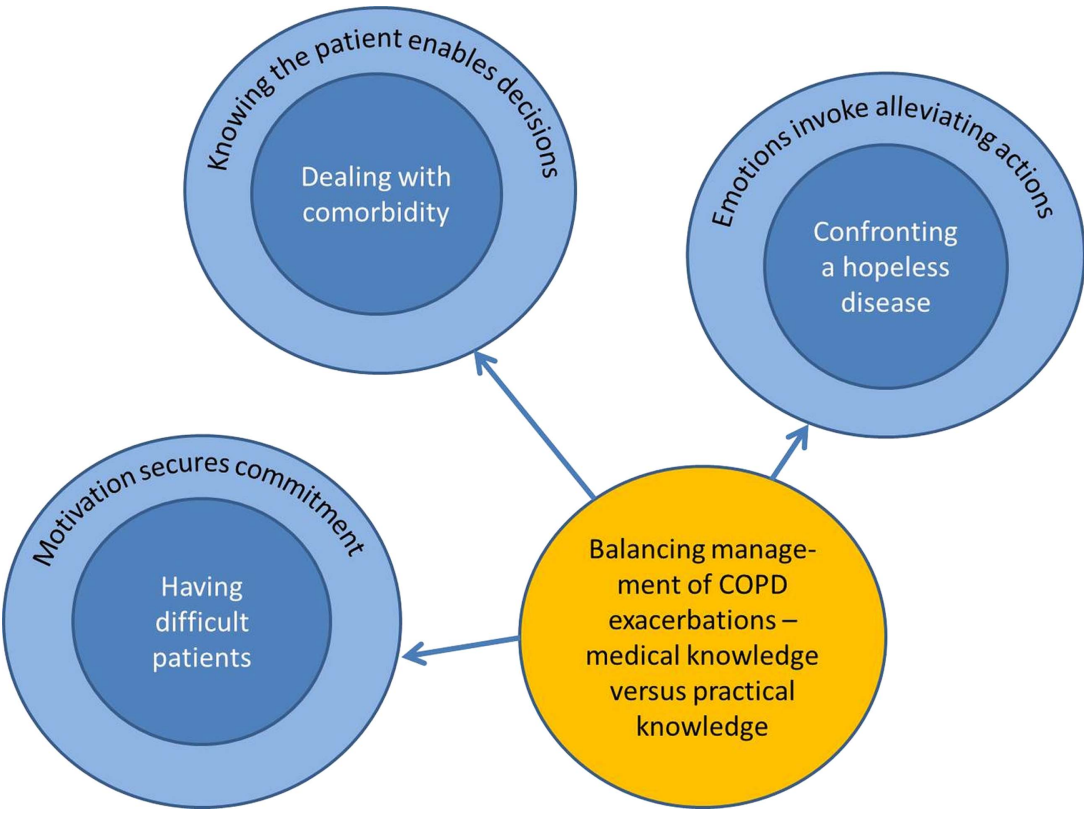

instructions to patients and to target the instructions according to their capacities-that is, mainly an approach to optimise knowledge. The GPs may need supportive actions for this from the health system and society. Concerning self-treatment, concrete future steps were identified in the data such as using management plans including 'rescue packs', having a nurse to take specific care of self-treatment and arranging teaching sessions involving the patient's spouse and family, as has shown to be effective in, for example, Bourbeau et al. ${ }^{32}$ However, as suggested by Abbot, the most fruitful approach might be to deal with 'the difficult interaction' rather than targeting either the patient or the physician. ${ }^{33}$ Therefore, programmes that specifically focus on improving the physician-patient relationship might be worth investigating.

Our finding that physicians feel powerless and frustrated because they have nothing really to offer the patient has been reported in other qualitative studies, ${ }^{34}$ but detailed literature on this subject is very limited. Physicians feel that they do their best, approach the patient with care and try to work according to the guidelines, but that there is little progress and only deterioration in the condition of the patient. Practical future steps include prioritising pulmonary rehabilitation, including adequate resourcing and ensuring that it is accessible for those in need, as well as a specific focus on physical exercise and physiotherapy. Also, a more concurrent focus on palliative needs and care and the ability to refer these patients to palliative teams were warranted. Pinnock et $a l^{35}$ suggest that an assessment should take place related to hospital admission for exacerbations. A patient study determining palliative needs found considerable needs in relation to breathlessness but fewer in the end-of-life stage. ${ }^{36}$ This is elaborated in Habraken $e t a l^{37}$ who point to the silence of patients with COPD about end-stage needs because they do not realise there are possibilities to improve their condition. This, together with our study of the physicians, suggests potentials for improvement of palliative care.

Overall, the physicians in this study refer in many different ways to the significance of knowing the patientand different dimensions of knowing him/her. We find that knowing concerns the disease and comorbidity and it addresses several practical issues of treatment: for example, a patient's difficulties using an inhaler, the support available from family and relatives, a patient's capacity to learn about colour codes for medicine or his ability to access a rehabilitation centre. In other words, knowing the patient also means that a GP does not or cannot always rest on clear evidence-based medicine, but that he, together with the patient, may deal with changing and context-dependent patient needs. We see this knowing in our data, where it shows that patient and physician together try to adapt to the best treatment and take into account situational contexts and practical and social circumstances. This approach is related to a concept promoted by Mol, 'the logic of care', which embraces the patient and the doctor as active parts who together create an adequate treatment. ${ }^{38}$ Knowing also is an approach that is central to the suggested collaborations between health professionals/health sectors and which is sought to be enhanced through collaboration.

The overall strength of this study is to be found in the design. It was designed as a cross-country study in order to attempt to find common crucial concerns within COPD exacerbation management in different health settings. There is a general lack of such comparative studies and our findings are grounded effectively in the whole empirical material. On the other hand, the focus on common issues may overshadow local contexts and local details on management. Also, there is always a danger that a comparative aim looks more for commonalities and convergences than for divergences. However, during 
the analysis we made an effort to scrutinise any major deviances to determine whether they had significance for the development of new analytical concepts or whether they were dimensions or properties to already found categories. Other variations are a part of the detailed examples of the illustrated concerns. Hong Kong was chosen to compare European management to a supposedly different kind of management, but the data from Hong Kong turned out to support and comply with the analysis of the other countries. A weakness in the study and this analysis is that we were not able to specify exactly what role the different health contexts played for the construction of the concerns. That is, we were aware that discussions in the FGDs were embedded in local health systems. They played a part in how physicians talked about their own medical practice, how their practices were framed economically and how working conditions were experienced and practically operationalised. Furthermore, GPs and respiratory physicians might not necessarily share the same medical knowledge background. However, a large part of the participants had changed career from, for example, GP to internal medicine, or working in hospital service for several years before becoming a GP. Also many worked in outpatient-policlinics (GPs and respiratory physicians) or outpatient-clinics combined with either hospital wards or health centres (GPs). This, we believe, lumps the two specialties together rather than splitting them concerning the medical experience. Hence, regarding the concerns we found in the analysis, it still seems justified to talk about shared concerns. But certainly, we fully acknowledge that there are differences within the concerns that are both culture-specific, health systemspecific and determined by different social and medical practices. We argue that the concerns are alike, but how to administer them in detail, how the specific perception was of patients, how collaboration could be improved, etc, varied from country to country. Future analyses of the dataset will draw in more focus on, for example, differences among the countries in self-treatment and the role of health systems. Methodologically, the fact that the FGDs were performed by different moderators did result in variations of moderation style and subsequent heterogeneity in FGDs. All were trained to perform alike with the same questioning route to follow but focus groups develop independently, often as a result of the participants. That the participants sometimes knew each other may have created a barrier for critical discussions, especially if seniors and juniors were together. It did, however, also in some cases, give a more safe setting for in-depth discussions. The moderators' background in general practice and interest in respiratory diseases may, on one hand, have been an advantage because the topics of the FGDs were well known. But, on the other hand, it might have created a blind eye towards specific areas. We saw that the PhD student and the epidemiologist more than others asked into themes just to understand what was meant. Also their background in different nationalities and medical discourses may have coloured the interviews, but this counts for the participants as well. Most importantly, all major topics were discussed in all FGDs and the methodological conditions above did not, in our view, jeopardise the present analysis. Concerning the translation there is always a risk that phrases and concepts have lost their significance during translation-certain translations were, however, discussed with the responsible researcher when the first author became in doubt about its content. Another limitation is that the interviews and the study overall were intended to focus on exacerbations, and while the patient history and the medical discussions did so, it proved inevitable for the physicians to leave out the thoughts and reflections on the patients with COPD and the COPD disease in general in many other aspects of the interviews. This is especially reflected in thoughts related to the third concern. We were aware of this during analysis and decided not to try splitting the results artificially into what dealt with exacerbations and what did not. Finally, sampling of the participants was intended to be strategic but turned out to be more pragmatic due to recruitment difficulties and due to differences in health systems.

Unanswered questions and future research derived from our study point to the need for more observational studies on how management in real life takes place. Studies addressing the benefits of management plans and understanding the low status of patients with COPD among GPs would also be of benefit.

\section{CONCLUSION}

Knowing the patient is essential in dealing with comorbidities as well as with difficult relations in the consultations on exacerbations. This study suggests that it is crucial to improve collaboration between primary and secondary care, in terms of, for example, shared consultations and defined work tasks, which may enhance shared knowledge of patients, medical decision-making and improved management planning. It also suggests that the GPs need supportive actions from the healthcare system and the society to target difficult consultations. Further studies are needed on barriers in the doctor-patient collaboration and how to reduce the GPs frustration with patients with COPD, in order to promote an optimistic and fruitful attitude to this group of patients.

\section{Author affiliations}

${ }^{1}$ Department of Community Medicine, General Practice Research Unit, UiT The Arctic University of Norway, Tromsø, Norway

${ }^{2}$ CAPHRI School for Public Health and Primary Care, Maastricht University, Maastricht, The Netherlands

${ }^{3}$ Department of Family and Community Medicine, Medical University of Lodz, Lodz, Poland

${ }^{4}$ Cochrane Institute of Primary Care and Public Health, School of Medicine, Cardiff University, Cardiff, UK

${ }^{5}$ Department of Family Medicine, University of Rostock, Rostock, Germany ${ }^{6}$ Department of Family Medicine, Northern State Medical University, Arkhangelsk, Russian Federation 
${ }^{7}$ The Jockey Club School of Public Health and Primary Care, The Chinese University of Hong Kong, Hong Kong, Hong Kong

Contributors MBR is the guarantor of the paper and she did the main analysis and interpretation of the data and drafted the article until final version. RI, MS, MG-C and NF performed FGDs and contributed to analysis and interpretation of the data. AA, EA and KK performed FGDs and HM contributed with overall conception of the study. All authors participated in the design of the study, revised the article critically for important intellectual content and gave final approval of the version to be published.

Funding This work was supported by the Research Council of Norway, grant number 202650//H10.

Ethics approval Wales: South East Wales Research Ethics Committee Panel C, Reference number: 11/WA/0049. The Netherlands: Medical Ethical Review Committee of University Hospital Maastricht/Maastricht University. Reference number is: MEC 11-05-025. Russia: Ethics Committee of Northern State Medical University (Troitsky 51, Arkhangelsk, Russia,163000), the number of approval N 23/12 from 8th of December 2010.

Provenance and peer review Not commissioned; externally peer reviewed.

Data sharing statement Additional unpublished data are available to all researchers who took part in the study. Every research centre has immediate access to data sampled in their own country and may have access to data from other countries if wished. These are all administered in a database at the General Practice Research Unit, University of Tromsø, by the project leader of the project PEXACO, and may be shared digitally on request.

Open Access This is an Open Access article distributed in accordance with the Creative Commons Attribution Non Commercial (CC BY-NC 3.0) license, which permits others to distribute, remix, adapt, build upon this work noncommercially, and license their derivative works on different terms, provided the original work is properly cited and the use is non-commercial. See: http:// creativecommons.org/licenses/by-nc/3.0/

\section{REFERENCES}

1. Melbye $H$, Joensen L, Risor MB, et al. Symptoms of respiratory tract infection and associated care-seeking in subjects with and without obstructive lung disease; the Tromso Study: Tromso 6. BMC Pulm Med 2012;12:51.

2. Zoia MC, Corsico AG, Beccaria M, et al. Exacerbations as a starting point of pro-active chronic obstructive pulmonary disease management. Respir Med 2005;99:1568-75.

3. Tinkelman DG, Price DB, Nordyke RJ, et al. Misdiagnosis of COPD and asthma in primary care patients 40 years of age and over. $J$ Asthma 2006;43:75-80.

4. Kuebler KK, Buchsel PC, Balkstra CR. Differentiating chronic obstructive pulmonary disease from asthma. J Am Acad Nurse Pract 2008;20:445-54.

5. Garcia-Aymerich J, Barreiro E, Farrero E, et al. Patients hospitalized for COPD have a high prevalence of modifiable risk factors for exacerbation (EFRAM study). Eur Respir $J$ 2000;16:1037-42.

6. Vestbo J, Hurd SS, Agusti AG, et al. Global strategy for the diagnosis, management, and prevention of chronic obstructive pulmonary disease: GOLD executive summary. Am J Respir Crit Care Med 2013;187:347-65.

7. Halpern MT, Stanford RH, Borker R. The burden of COPD in the USA: results from the Confronting COPD Survey. Respir Med 2003;97(Suppl C):S81-9.

8. Decramer M, Nici L, Nardini S, et al. Targeting the COPD exacerbation. Respir Med 2008;102(Suppl 1):S3-15.

9. Anzueto A. Primary care management of chronic obstructive pulmonary disease to reduce exacerbations and their consequences. Am J Med Sci 2010;340:309-18.

10. Arne M, Emtner M, Janson S, et al. COPD patients perspectives at the time of diagnosis: a qualitative study. Prim Care Respir J 2007;16:215-21.

11. Jones RC, Hyland ME, Hanney $\mathrm{K}$, et al. A qualitative study of compliance with medication and lifestyle modification in Chronic
Obstructive Pulmonary Disease (COPD). Prim Care Respir J 2004;13:149-54.

12. Harris $\mathrm{D}$, Hayter M, Allender S. Improving the uptake of pulmonary rehabilitation in patients with COPD: qualitative study of experiences and attitudes. Br J Gen Pract 2008;58:703-10.

13. Coleman T, Cheater F, Murphy E. Qualitative study investigating the process of giving anti-smoking advice in general practice. Patient Educ Couns 2004;52:159-63.

14. Cleland J, Thomas M, Pinnock $\mathrm{H}$. The views and attitudes of general practitioners and smokers toward provision of smoking cessation advice: a qualitative study. Prim Care Respir $J$ 2004;13:144-8.

15. Jacot S I, Ruffieux C, Cornuz J. Self-reported smoking cessation activities among Swiss primary care physicians. BMC Fam Pract 2009;10:22

16. Vogt F, Hall S, Marteau TM. General practitioners' and family physicians' negative beliefs and attitudes towards discussing smoking cessation with patients: a systematic review. Addiction 2005;100:1423-31.

17. Walters JA, Hansen EC, Walters EH, et al. Under-diagnosis of chronic obstructive pulmonary disease: a qualitative study in primary care. Respir Med 2008;102:738-43.

18. Walters JA, Hansen EC, Johns DP, et al. A mixed methods study to compare models of spirometry delivery in primary care for patients a risk of COPD. Thorax 2008;63:408-14

19. Crawford A. Respiratory practitioners' experience of end-of-life discussions in COPD. Br J Nurs 2010;19:1164-9.

20. Blumer H. Symbolic interactionism: perspective and method. Prentice Hall, 1969

21. Hood JC. Orthodoxy vs. power: the defining traits of grounded theory. In: Bryant A, Charmaz K. eds The Sage handbook of grounded theory. Sage Publications, 2007:151-64.

22. Corbin J, Strauss A. Basics of qualitative research. Techniques and procedures for developing grounded theory. Sage Publications, 2008.

23. Charmaz K. Constructing grounded theory. A practical guide through qualitative analysis. Sage Publications, 2006.

24. Barbour RS. Focus groups. In: Bourgeault I, Dingwal R, de Vries R, eds. The Sage handbook of qualitative methods in health research. Sage Publications, 2010:327-53

25. Krueger RA, Casey MA. Focus groups. A practical guide for applied research. Sage Publications, 2009.

26. Tsiligianni IG, Kosmas E, Van der Molen T, et al. Managing comorbidity in COPD: a difficult task. Curr Drug Targets 2013;14:158-76.

27. Guthrie B, Payne K, Alderson P, et al. Adapting clinical guidelines to take account of multimorbidity. BMJ 2012;345:e6341.

28. Halding AG, Heggdal K, Wahl A. Experiences of self-blame and stigmatisation for self-infliction among individuals living with COPD. Scand J Caring Sci 2011;25:100-7.

29. Berger BE, Kapella MC, Larson JL. The experience of stigma in chronic obstructive pulmonary disease. West J Nurs Res 2011;33:916-32.

30. Sheridan N, Kenealy T, Salmon E, et al. Helplessness, self blame and faith may impact on self management in COPD: a qualitative study. Prim Care Respir J 2011;20:307-14, 1.

31. Fiester A. The "difficult" patient reconceived: an expanded moral mandate for clinical ethics. Am J Bioeth 2012;12:2-7.

32. Bourbeau J, Julien M, Maltais F, et al. Reduction of hospital utilization in patients with chronic obstructive pulmonary disease: a disease-specific self-management intervention. Arch Intern Med 2003;163:585-91.

33. Abbott J. Difficult patients, difficult doctors: can consultants interrupt the "blame game"? Am J Bioeth 2012;12:18-20.

34. Lundh L, Rosenhall L, Tornkvist L. Care of patients with chronic obstructive pulmonary disease in primary health care. $J$ Adv Nurs 2006;56:237-46.

35. Pinnock H, Kendall M, Murray SA, et al. Living and dying with severe chronic obstructive pulmonary disease: multi-perspective longitudinal qualitative study. BMJ 2011;342:d142.

36. White $\mathrm{P}$, White $\mathrm{S}$, Edmonds $\mathrm{P}$, et al. Palliative care or end-of-life care in advanced chronic obstructive pulmonary disease: a prospective community survey. Br J Gen Pract 2011;61:e362-70.

37. Habraken JM, Pols J, Bindels PJ, et al. The silence of patients with end-stage COPD: a qualitative study. $\mathrm{Br} J$ Gen Pract 2008;58:844-9.

38. Mol A. The logic of care-health and the problem of patient choice. Routledge, 2008. 